\title{
Persistence of myelofibrosis treated with ruxolitinib: biology and clinical implications
}

\author{
David M. Ross, ${ }^{1,2,3}$ Jeffrey J. Babon, ${ }^{4}$ Denis Tvorogov ${ }^{2}$ and Daniel Thomas ${ }^{3}$ \\ ${ }^{1}$ Department of Hematology and Bone Marrow Transplantation, Royal Adelaide Hospital, \\ Adelaide; ${ }^{2}$ Centre for Cancer Biology, University of South Australia and SA Pathology, \\ Adelaide; ${ }^{3}$ Precision Medicine Theme, South Australian Health \& Medical Research \\ Institute, and Adelaide Medical School, University of Adelaide, Adelaide and ${ }^{4}$ The Walter \\ and Eliza Hall Institute of Medical Research and Department of Medical Biology, \\ University of Melbourne, Parkville, Australia.
}

Haematologica 2021

Volume 106(5):1244-1253

\section{ABSTRACT}

\begin{abstract}
$\Lambda$ ctivation of JAK-STAT signaling is one of the hallmarks of myelofibrosis, a myeloproliferative neoplasm that leads to inflammation, progressive bone marrow failure, and a risk of leukemic transformation. Around $90 \%$ of patients with myelofibrosis have a mutation in JAK2, MPL, or CALR: so-called 'driver' mutations that lead to activation of JAK2. Ruxolitinib, and other JAK2 inhibitors in clinical use, provide clinical benefit but do not have a major impact on the abnormal hematopoietic clone. This phenomenon is termed 'persistence', in contrast to usual patterns of resistance. Multiple groups have shown that type 1 inhibitors of JAK2, which bind the active conformation of the enzyme, lead to JAK2 becoming resistant to degradation with consequent accumulation of phospho-JAK2. In turn, this can lead to exacerbation of inflammatory manifestations when the JAK inhibitor is discontinued, and it may also contribute to disease persistence. The ways in which JAK2 V617F and CALR mutations lead to activation of JAK-STAT signaling are incompletely understood. We summarize what is known about pathological JAK-STAT activation in myelofibrosis and how this might lead to future novel therapies for myelofibrosis with greater disease-modifying potential.
\end{abstract}

\section{Introduction}

Primary myelofibrosis is a peculiar illness that has features of both a slowly progressive cancer and a chronic inflammatory disorder. It is a clonal neoplasm driven by a handful of somatic mutations that activate cell signaling, presumably residing in the long-term stem cell compartment, ${ }^{1-3}$ but it also has a constellation of cytokine-mediated symptoms that are disproportionately severe. Myelofibrosis can also arise from antecedent polycythemia vera or essential thrombocythemia, leading to substantially overlapping clinical features. Progression of myelofibrosis leads to bone marrow failure with extensive marrow fibrosis or transformation to secondary acute myeloid leukemia. After the discovery of activating JAK2 mutations in $50-60 \%$ of patients with primary myelofibrosis, ${ }^{4,5}$ clinicians were hopeful that myelofibrosis would respond to tyrosine kinase inhibitor (TKI) therapy in a similar fashion to chronic myeloid leukemia, another myeloproliferative neoplasm (MPN). Chronic myeloid leukemia is driven by the BCR-ABL1 fusion which responds to ABL1 TKI therapy with rapid log-fold reductions in the number of BCR-ABL1-mutated cells in the majority of patients. ${ }^{6.8}$ Ruxolitinib was the first dual JAK1/JAK2 TKI approved for the treatment of myelofibrosis and was demonstrated to reduce splenomegaly and improve many symptoms related to myelofibrosis but, unlike TKI treatment in chronic myeloid leukemia, it does not result in elimination of the mutant clonal population of cells (a phenomenon termed "disease persistence" in contrast to the conventional understanding of TKI resistance) nor does it cause widespread regression of fibrosis. Understanding the molecular basis of clinical responses to ruxolitinib is of great relevance to cancer biology and has implications for prescribing (and stopping) therapy, as well as the future design of kinase inhibitors for other cancers. Here we review what is known to date about the 
mechanism of ruxolitinib persistence, its relationship to various recurrent somatic mutations in myelofibrosis, and potential ways to circumvent persistence to improve outcomes.

\section{Physiological JAK-STAT signaling}

JAK family kinases are non-receptor tyrosine kinases that are crucial for signal transduction of many cytokines and growth factors. The family comprises four members: JAK1, JAK2, JAK3, and TYK2. . JAK family kinases are preassociated with the cytoplasmic portion of their cognate receptors via their FERM and SH2 domains. Cytokineinduced receptor dimerization facilitates JAK kinase transactivation and phosphorylation of tyrosine residues in the activation loop, as well as local phosphorylation of receptor cytoplasmic-tail tyrosine residues and tyrosine residues on associated signaling molecules. JAK2 contains a carboxy-terminal JAK homology domain, JH1, which has tyrosine kinase activity and transfers ATP to a protein substrate (such as STAT3 or STAT5), together with a JH2 pseudokinase domain, which is believed to regulate the activity of $\mathrm{JH} 1$.

Uncontrolled signaling by JAK2 is prevented by at least three major negative regulatory mechanisms. Activation loop phosphorylation of Tyr1007/1008 can be removed by tyrosine phosphatases including PTP1B, TC-PTP, SHP1, SHP2, CD45, and PTP-RT. Secondly, SOCS proteins (SOCS1-7 and CIS) are transcriptionally upregulated following receptor activation and provide negative feedback loops that restrict the duration of active signaling by either directly inhibiting JAK or by promoting the degradation of the associated cytokine receptor. ${ }^{10-13}$ Thirdly, phosphorylated JAK2 (p-JAK2) is ubiquitinated by CBL-family E3 ubiquitin ligases, ${ }^{14}$ as well as SOCS1, leading to proteasomal degradation, normally within minutes of receptor activation. Ubiquitination is itself a reversible process mediated by de-ubiquitinases, such as USP9X. ${ }^{15}$

\section{Molecular aspects of JAK2 and ruxolitinib}

The commonest somatic mutation in myelofibrosis is $J A K 2$ V617F, which is present in $50-60 \%$ of patients with primary myelofibrosis and post-essential thrombocythemia myelofibrosis, and in $95 \%$ of patients with myelofibrosis following polycythemia vera. ${ }^{16}$ The V617F point mutation has been shown to disrupt the normal auto-inhibitory function of the JH2 domain leading to dysregulated activation of JAK-STAT signaling which, both in animal models and in patients, contributes to many of the cardinal manifestations of the disease. Other mutations that occur in MPN may activate a cytokine receptor (e.g., thrombopoietin receptor) or downstream signaling proteins, including NRAS, KRAS, and PTPN11. The homodimeric type 1 receptor for thrombopoietin regulates platelet formation and is encoded by MPL.

The exact molecular mechanism of JAK2 V617F, in comparison with the action of wild-type JAK2 and other kinases such as JAK1 or TYK2, is still being elucidated. The molecular signature of this mutation is that induces JAK autophosphorylation (activation) in the absence of cytokine. In vitro the presence of a homodimeric cytokine receptor is necessary for this to occur. ${ }^{17,18}$ Interestingly, the
JH2 domain of mutated JAK2 in myelofibrosis lacks an Asp in the His/Arg/Asp motif of its catalytic loop and possesses no definitive kinase activity, even though it is required for cytokine receptor activation. The JH2 domain has, however, been shown to phosphorylate two negative regulatory residues of JAK2 (Ser523 and Tyr570), which may contribute to the increased kinase activity. ${ }^{19}$ A biochemical study showed that the orthologous mutation in JAK1 (JAK1 V658F, which has been found in patients with acute lymphoblastic leukemia and confers cytokine-independence in transduced $\mathrm{Ba} / \mathrm{F3}$ cells ${ }^{20,21}$ ) does not enhance the catalytic activity of the isolated kinase or indeed any other measurable enzymatic parameter. ${ }^{10}$ These findings are all consistent with the hypothesis that the V617F mutation does not render JAK2 more "active" when switched on but rather leads to its being switched on in inappropriate circumstances, such as in the absence of cytokine. Consistent with this, it was shown recently that JAK2 V617F promotes cytokine-independent receptor dimerization, thereby activating the kinase in the absence of an appropriate signal. ${ }^{22}$ Understanding the exact molecular effects of V617F will be important in the design of V617F-specific therapies that have lower activity against wild-type JAK2. Emerging in vitro and clinical data suggest that various JAK-STAT pathway-activating mutations (JAK2, MPL, SH2B3, NRAS, KRAS, PTPN11), as well as mutations in the protein scaffold CALR, all activate JAKSTAT signaling by subtly distinct mechanisms. This may have implications for treatment outcomes and suggests the existence of mutation-specific differences in ruxolitinib sensitivity and mechanisms of persistence.

More than $90 \%$ of cases of myelofibrosis show mutational evidence of JAK-STAT activation, ${ }^{16}$ suggesting that this pathway is a critical "necessary" driver of the pathology. However, the converse is not true: having a mutation in the JAK-STAT pathway does not inevitably lead to myelofibrosis, i.e., JAK2 V617F or mutant MPL is not sufficient for the disease phenotype. This is perhaps best exemplified by polycythemia vera, in which the JAK2 V617F variant allele frequency is commonly in the range $50-100 \%,{ }^{23}$ yet progression to myelofibrosis occurs in only $20-30 \%$ of individuals. This risk is time-dependent and was $15 \%$ with a median follow-up of 8 years in one study. ${ }^{24}$ Emerging studies comprehensively detailing the genetic landscape highlight that myelofibrosis is a multi-mutation disease in the majority of patients. ${ }^{16}$ Importantly, mutations in epigenetic genes such as EZH2, ASXL1 or splicing factors as well the presence of specific inflammatory cytokines may link to fibrotic aspects of the pathology. ${ }^{25}$ In a large cohort of MPN patients, Grinfeld and colleagues performed an analysis to identify mutations that were associated with myelofibrosis versus chronic phase MPN. Five of the six genes with the highest odds ratio for myelofibrosis were epigenetic regulators (ZRSR2, U2AF1, SRSF2, EZH2, and ASXL1) and all had an odds ratio higher than that for JAK2 at an allele burden $>50 \%{ }^{16}$ Although some of these mutations are likely acquired during disease evolution, there is also evidence that epigenetic changes early in MPN development may affect the phenotype. In a few patients with MPN who had mutations in both TET2 (which regulates DNA methylation) and JAK2, the progenitor cells that appeared to have acquired TET2 mutations first were shown to be less sensitive to ruxolitinib than progenitors from other patients in whom JAK2 was acquired first. ${ }^{26}$ Determining the epigenetic mechanisms that contribute to ruxolitinib persistence/ 
resistance is an exciting area of ongoing research that is beyond the scope of this review.

\section{Molecular aspects of CALR and ruxolitinib}

Somatic mutations in exon 9 of the gene coding for the endoplasmic chaperone protein calreticulin $(C A L R)$ are found in 70-80\% of patients with JAK2-negative myelofibrosis and account for $\sim 30 \%$ of myelofibrosis cases overall. ${ }^{2,27}$ Virtually all CALR mutations in MPN are small insertions or deletions clustered in exon 9, resulting in a +1 frameshift and loss of the last four amino acids (KDEL) that form the endoplasmic reticulum retention signal, leading to altered distribution of CALR. The two commonest mutations are a 52 bp deletion (type 1, which is present in $45-53 \%$ of patients) and a 5 bp insertion (type 2 , present in $32-41 \%$ of patients). Although more than 50 $C A L R$ mutations have been described, the majority can be classified as type 1-like or type 2-like based on bioinformatic predictions of protein structure. ${ }^{28}$ This functional classification is clinically relevant as it influences prognosis in myelofibrosis. ${ }^{29,30}$ The reason for differing prognosis is unclear, but there are biological differences that might be relevant: type 1 mutations eliminate all the negative charge of the C-terminal domain eliminating its calcium binding, thus potentially activating proteases and protein misfolding in the endoplasmic reticulum, ${ }^{31}$ and potentially altering the chaperone function of CALR.

Data from independent laboratories have shown that mutant CALR protein requires MPL for signaling and factor-independent cell growth, and that the normal lectin domain of CALR is essential to bind glycosylated sites on MPL. ${ }^{32-34}$ More recently, it was reported that both mutant and wild-type CALR proteins are present at higher levels in the plasma of myelofibrosis patients (compared to normal individuals), ${ }^{35,36}$ and may function in a paracrine fashion by binding the extracellular domains of MPL to facilitate receptor dimerization. ${ }^{34,36}$ However, the relative contribution of autocrine versus paracrine versus endosomal signaling of mutant CALR protein to aberrant activation of the JAK-STAT pathway in MPN has not been fully elucidated (Figure 1).

As stated, activating mutations in the juxtamembrane domain of $M P L$ are found in $5-10 \%$ of patients with myelofibrosis. Both CALR and MPL mutations presumably signal through JAK2, and there is evidence of therapeutic benefit from ruxolitinib in experimental models and in patients with these mutations. In these cases, the JAK inhibitor is binding wild-type JAK2, so there is limited selectivity of the TKI for MPN cells. Consistent with this, patients with JAK2-negative myelofibrosis may have slightly inferior clinical responses to ruxolitinib (discussed more fully below) but, because of the relatively small number of such patients in the COMFORT studies, additional studies are needed to confirm this. ${ }^{37}$

\section{Ruxolitinib is a "type I" ATP-competitive inhibitor}

Historically, pan-JAK inhibitors (such as AG-490) were first developed as molecules that were substrate-competitive for tyrosine residues, and either mixed competitive or non-competitive for ATP. In recent years, drug devel- opment has focused on ATP-competitive inhibitors, such as ruxolitinib. All clinically approved JAK inhibitors (ruxolitinib, fedratinib) bind and stabilize the kinase-active conformation of JAK2, and are known as "type I" inhibitors, in contrast to "type II" ATP-competitive inhibitors which bind and stabilize the protein in its inactive conformation. Exactly how these varying binding mechanisms play out in the clinic and their association with susceptibility to disease persistence are exciting areas of ongoing research.

JAK2 V617F mutations are remarkable among recurrent oncogenic single nucleotide variants reported in the Catalogue of Somatic Mutations In Cancer (COSMIC) for having a consistent inflexible substitution, namely replacement of a small hydrophobic by a large hydrophobic residue outside the catalytic domain, generally implying a "change in function" rather than simply a loss or non-specific gain in function. Notably, the mutated JH2 pseudokinase domain does not bind ruxolitinib directly. This is in contrast to activating point mutations in FLT3 (such as D835Y, D835H, F691L) or gate-keeper mutations

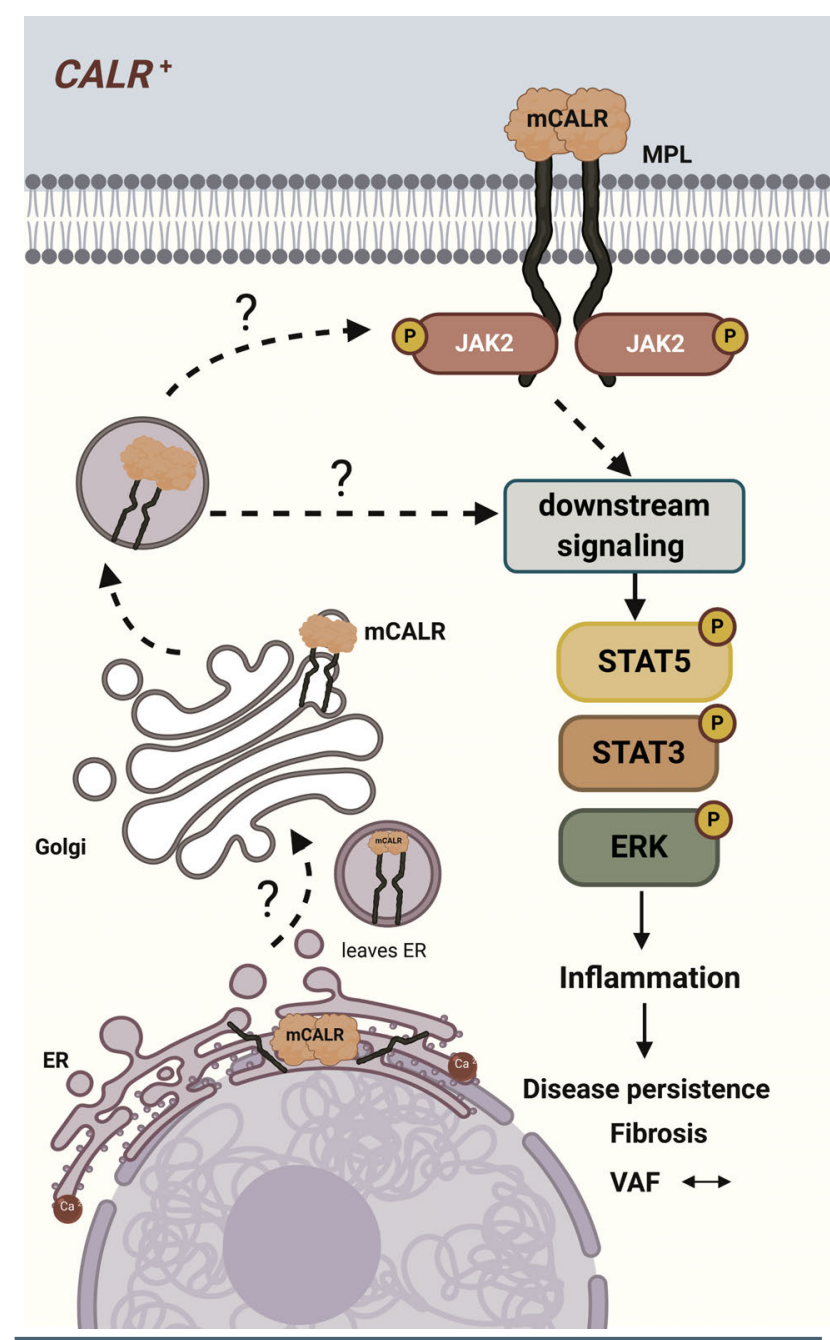

Figure 1. Activation of JAK-STAT signaling in CALR-mutant cells is inhibited by ruxolitinib. Schematic representation of mutant CALR (mCALR) trafficking in MPN cells showing inhibition by ruxolitinib. The exact mechanism(s) by which mCALR activates JAK-STAT signaling are still being elucidated. Mutant CALR leaves the endoplasmic reticulum and is associated with MPL to promote homodimerization and activation of JAK2. 
found in $A B L 1$, which involve a range of substitutions and directly alter binding of the drug with the enzyme.

\section{What are the long-term outcomes for patients treated with ruxolitinib?}

The COMFORT-1 study compared ruxolitinib with placebo for the treatment of myelofibrosis, ${ }^{38}$ and the COMFORT-2 study compared ruxolitinib with best available therapy, including supportive care, hydroxyurea, and a range of other medical therapies..$^{39}$ The primary endpoint in both studies was the proportion of patients achieving a $35 \%$ or greater reduction in spleen volume. The proportion of patients who achieved this response after 24 weeks of ruxolitinib treatment was $42 \%$ in COMFORT-1 and $32 \%$ in COMFORT-2. ${ }^{38,39}$ The response rates in the respective comparator arms of the two studies were $0.7 \%$ and $0 \%$.

In a subsequent analysis of a subset of patients from the COMFORT-2 study the response rate after 48 weeks was $20 \%$ (confidence interval: $5.7-43.7 \%$ ) in 20 patients with a CALR mutation versus $34 \%$ (confidence interval: 24.8$44.1 \%$ ) in 100 CALR-negative patients, most of whom had a JAK2 V617F mutation. ${ }^{37}$ Overall, there was no statistically significant difference in splenic response rate or survival between patients with and without JAK2 mutations, but the JAK2-positive patients had a numerically greater reduction in spleen size ${ }^{40} J A K 2$ V617F is almost universally present in post-polycythemia vera myelofibrosis, which is characterized by loss of heterozygosity and high allelic burden, ${ }^{41}$ and can perhaps be considered to epitomize JAK2-driven myelofibrosis. In this subgroup the hazard ratio for overall survival was 0.25 , compared with 0.65 in primary myelofibrosis. ${ }^{40}$ Whether this reflects the higher frequency of high-risk genomic lesions in primary myelofibrosis or 'on-target' efficacy through inhibition of JAK2 V617F remains to be established. Overall, these clinical data emphasize that ruxolitinib is not a JAK2 mutant-specific therapy, although patients with CALR mutations might have mildly inferior responses.

\section{Does ruxolitinib eradicate cells containing disease-causing driver mutations?}

Although ruxolitinib is now established as the standard therapy for symptomatic myelofibrosis, evidence of a long-term effect on disease biology is limited. The average reduction in JAK2 allelic burden was $7-22 \%$ after 48 weeks of treatment in evaluable patients; ${ }^{38,42}$ regression of fibrosis in the marrow was seen in around $16 \%$ of patients at last follow-up (median 2.2 years), ${ }^{43}$ and there was no change in the risk of leukemic transformation. Cases of complete hematologic or molecular remission have been reported, ${ }^{44}$ but are very uncommon. Typically there is a gradual loss of response over time, with approximately $27 \%$ of patients remaining on first-line ruxolitinib treatment after 5 years in the COMFORT studies. In the subgroup of patients who achieved a $35 \%$ or greater reduction in spleen volume, the median duration of the response was 3.2 years. ${ }^{43,45}$

The immunophenotype of myelofibrosis-initiating cells has not been well studied, principally because of a lack of robust engraftment models, but in a few patients analyzed carefully, the stem cell population appeared to reside within the $\mathrm{CD} 34^{+} \mathrm{CD} 388^{-} \mathrm{CD} 90^{+}$compartment, as determined using a humanized bone marrow niche. ${ }^{3}$ Flow cytometry studies suggest a high level of circulating $\mathrm{CD} 34^{+} \mathrm{CD} 38^{-}$ hematopoietic stem-like cells in patients with mutant CALR, but all these cells were resistant to ruxolitinib in colony assays. New models of myelofibrosis will help to characterize the disease-propagating stem-like cells and identify critical cell surface markers for monitoring future disease-modifying therapies.

\section{What are the possible mechanisms of ruxolitinib persistence?}

A number of models have been proposed to explain ruxolitinib persistence, i.e., the ongoing outgrowth of mutated cells despite JAK-STAT pathway sensitivity to ruxolitinib, with varying levels of evidence. These include activation of alternative kinases not inhibited by ruxolitinib ${ }^{46}$ epigenetic mutations leading to growth advantage, ${ }^{26,47}$ phosphatase negative feedback inhibition (as noted with the failure of RAFK inhibitors in melanoma), ${ }^{48}$ lack of specificity for JAK2 V617F versus wild-type JAK2, ${ }^{10}$ and accumulation of p-JAK2 during exposure to ruxolitinib. The last of these phenomena is the most studied, with multiple lines of evidence across several independent studies suggesting that it is a contributory mechanism in clinically observed ruxolitinib persistence. ${ }^{49.51}$ Various therapeutic strategies have been proposed in addition to targeting JAK2 (reviewed by Bankar and Gupta ${ }^{52}$ ). Here we focus on the evidence regarding persistent activation of JAK-STAT signaling and its downstream proteins, and how these phenomena may be targeted by current or emerging therapies.

\section{Why does phosphorylation of JAK2 increase during ruxolitinib treatment?}

Initial laboratory studies studying ruxolitinib activity in vitro noted that, paradoxically, phosphorylation of JAK2 on Tyr $1007 / 1008$ located in the activation loop was observed at increased levels in V617F-positive cells following prolonged exposure to ruxolitinib. ${ }^{49}$ Both the total level of phosphorylation and the total amount of JAK2 protein were increased. ${ }^{49}$ Similar observations were also made for the type I inhibitor "JAK inhibitor 1" and Go6976, a protein kinase C inhibitor with potent activity against JAK2. ${ }^{53,54}$ Accumulation of p-JAK2 was noted to occur in the presence of total blockade of kinase function and inhibition of STAT and ERK phosphorylation downstream (Figure 2). It was noted that ruxolitinib-induced phosphorylation of JAK2: (i) was staurosporine-sensitive and ATP-dependent; (ii) required cytokine receptor interaction and intact JH1, FERM and JH2 domains; and (iii) could occur in V617F+ SET2 cells in the absence of JAK1 and TYK $2 .{ }^{50}$ In contrast, JAK2 wild-type cells (such as TF1 cells) showed little or no type I inhibitor-induced loop phosphorylation after growth factor starvation. A carboxyterminal-directed antibody could not immunoprecipitate JAK2 after exposure to type I inhibitor. All these findings are consistent with a conformational change in the kinase domain generated by ruxolitinib. Because of presumed structural flexibility, high resolution crystallography data are not available for the activation loop during type I inhibitor binding but, critically, activation of downstream STAT phosphorylation could be repro- 
ducibly detected following removal of the drug. This implies that the accumulated p-JAK could act as a pathological signaling node as the drug level falls in patients if drug is abruptly stopped or a dose missed.

More recently, Tvorogov and co-authors extended these findings to show that accumulation of p-JAK2 is due to resistance of $\mathrm{p}$-JAK2 to ubiquitination and degradation while bound to ruxolitinib. ${ }^{51}$ Specifically, immunoprecipitation with an anti-p-JAK2 antibody produced a band for p-JAK2 in untreated cells, but not in ruxolitinib-treated cells, consistent with an altered conformation induced by the drug. Accordingly, it was noted that cells exhibiting delayed phosphorylation kinetics (i.e., those with wildtype JAK2) were significantly more sensitive to ruxolitinib cytotoxicity and growth inhibition than cells with rapid phosphorylation kinetics (cells with JAK2 V617F). This was an important observation that raised the possibility that ruxolitinib allows drug-bound JAK protein to escape the negative feedback loops of dephosphorylation and degradation (Figure 2B).

To test this, recombinant JAK2 kinase was treated with a phosphatase in the presence of ruxolitinib. Ruxolitinib blocked the dephosphorylation by PTP1B for up to $20 \mathrm{~h}$ whereas reduction of Tyr1007/1008 phosphorylation normally occurred within $2 \mathrm{~h}^{.1}$ In keeping with this, ruxolitinib prevented any detectable ubiquitination of JAK2 after cytokine stimulation. These results suggested that binding of ruxolitinib induces a conformational change that conceals Tyr1007/1008 from phosphatase access, and ruxolitinib-bound p-JAK2 is no longer susceptible to dephosphorylation or degradation.

Interestingly, primary $C A L R$-mutant cells did not exhibit either JAK2 phosphorylation in the presence of ruxolitinib or striking withdrawal signaling to the same degree as the $J A K 2$ V617F samples. CALR-mutant myelofibrosis cells showed undetectable levels of activated JAK2 Tyr1007/1008 phosphorylation in the presence of ruxolitinib and in some CALR ${ }^{+}$samples and in a CALR-mutated cell line (MARIMO) total JAK2 protein was difficult to detect, if not completely absent. This is consistent with a number of emerging reports using CALR-mutated models of myelofibrosis ${ }^{55-57}$ suggesting fundamental differences in the nature of JAK activation in myelofibrosis patients with mutated CALR (Figure 1).

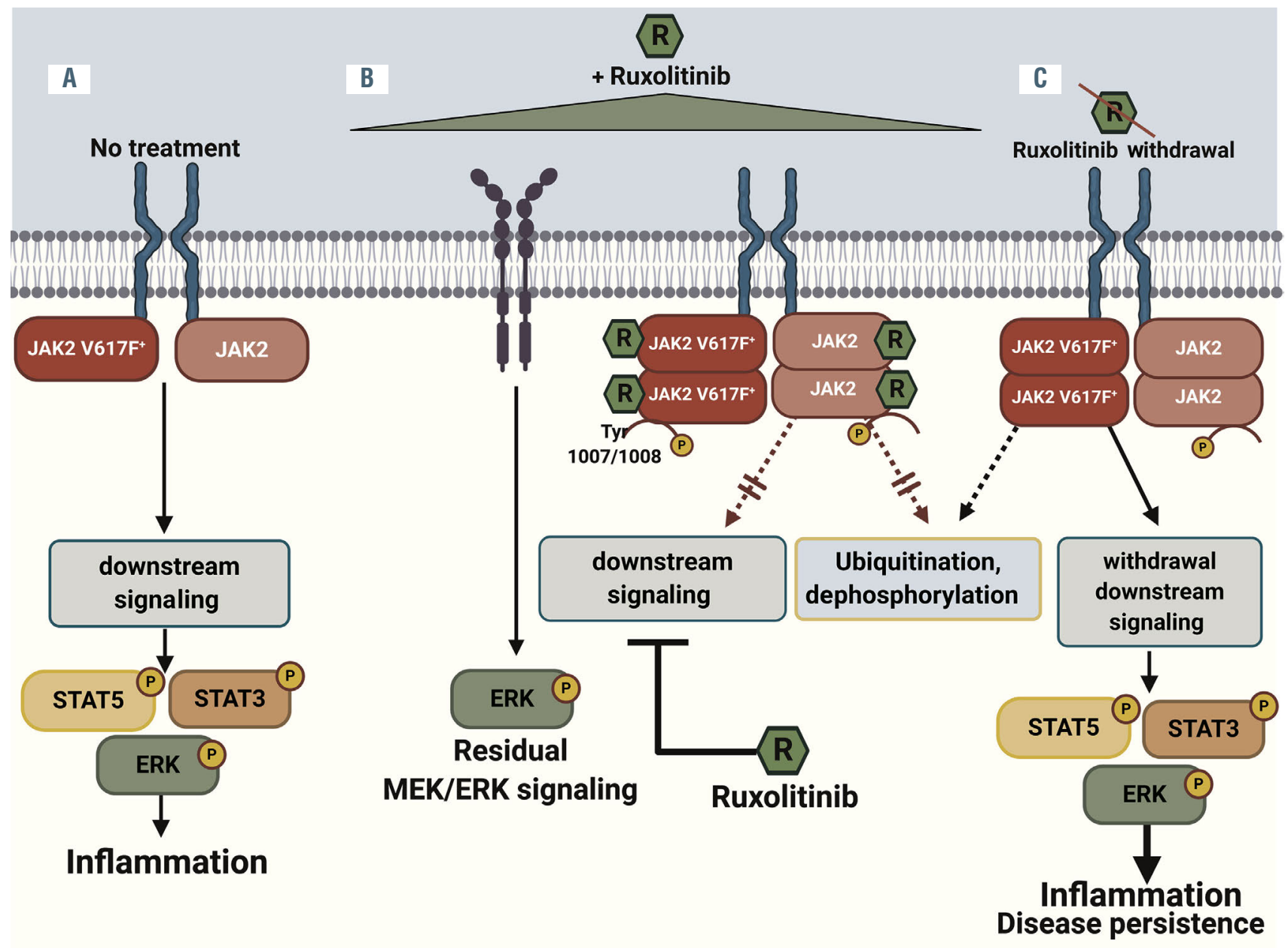

Figure 2. Signaling in JAK2 V617F cells before, during, and after discontinuation of ruxolitinib. Schematic representation of JAK-STAT activation in JAK2 V617F myeloproliferative neoplastic cells. (A) JAK2 V617F leads to increased signaling through STAT5/STAT3/ERK leading to proliferation and inflammation. (B) In the presence of a type 1 JAK2 inhibitor, such as ruxolitinib, signaling through the JAK-STAT pathway is abrogated, but so too is ubiquitination and degradation of JAK2, leading to accumulation of p-JAK2. Signaling through MEK/ERK remains activated and contributes to disease persistence. (C) Ruxolitinib discontinuation leads to transiently increased signaling through the accumulated pool of p-JAK2 with inflammatory symptoms. 


\section{Lessons from the clinic}

\section{Ruxolitinib discontinuation syndrome}

Interruption of ruxolitinib treatment typically leads to recrudescence of cytokine-mediated symptoms within a week ${ }^{38}$ often accompanied by increasing spleen size, highlighting the persistence of the underlying disease. The accumulation of p-JAK2 likely accounts for the prompt onset of withdrawal symptoms when ruxolitinib is stopped, since the increased p-JAK2 will rapidly lead to downstream signaling in the absence of the drug. Ruxolitinib has a short half-life of approximately $3 \mathrm{~h}{ }^{58}$ so the drug is washed out rapidly if dosing is interrupted. Although the accumulation of p-JAK2 is likely to be a class effect of type 1 JAK inhibitors, we are not aware of any reports of discontinuation syndrome with fedratinib, momelotinib, or pacritinib. Both fedratinib and pacritinib have very long half-lives of more than 24 h. ${ }^{59,60}$ Delayed clearance of these drugs may reduce the risk of withdrawal phenomena. Momelotinib has a half-life of $4-6 \mathrm{~h},{ }^{61}$ similar to that of ruxolitinib, so withdrawal phenomena might be predicted to occur with abrupt discontinuation of momelotinib.

In most patients who discontinue ruxolitinib there is a return to near baseline severity of symptoms, but in rare cases there are life-threatening manifestations thought to be due to an exaggerated inflammatory response. Ruxolitinib discontinuation syndrome is a diagnosis of exclusion based on a temporal relationship between drug withdrawal and onset of clinical manifestations, which can appear from less than $24 \mathrm{~h}$ to up to 3 weeks after discontinuation. In the original phase I/II trial, three of 47 patients who discontinued ruxolitinib developed acute respiratory distress syndrome. ${ }^{62}$ In the phase III COMFORT-I study, one patient developed acute respiratory distress, pyrexia and splenic infarction following ruxolitinib discontinuation..$^{38}$ Coltro and co-workers described a case of JAK2 V617F essential thrombocythemia evolving to acute myeloid leukemia with acute respiratory distress syndrome developing after ruxolitinib was discontinued, and improving within $48 \mathrm{~h}$ of re-introduction of the drug. ${ }^{63}$ Other reports include a patient who developed tumor lysis-like syndrome,$^{64}$ a case of acute respiratory failure, ${ }^{65}$ and a case of acute respiratory distress syndrome that twice resolved after ruxolitinib re-introduction. ${ }^{6}{ }^{6}$

Of note, of the nine published cases of life-threatening ruxolitinib discontinuation syndrome reported in the literature, all were reported to have a JAK2 mutation. This is a disproportionate enrichment compared to the prevalence of the CALR mutation ( $35 \%$ of cases of myelofibrosis). Further research into mutation-specific side effects of kinase inhibitors is warranted.

Some experts recommend tapering the ruxolitinib dose rather than abrupt discontinuation, especially when the reason for the therapy interruption is an adverse event other than cytopenia. ${ }^{6,68}$ One of the commonest causes of ruxolitinib discontinuation is infection. ${ }^{69}$ By stabilizing the active conformation of JAK2 in a manner that prevents dephosphorylation and degradation, type I inhibitors may rarely induce a signaling state that heuristically resembles a cytokine storm (Figure 2C). ${ }^{51}$ Experimental co-culture of JAK2 V617F cells with increased levels of cytokines in the medium promoted the accumulation of p-JAK2, since signaling through cytokine receptors is still capable of inducing p-JAK2 in the presence of ruxolitinib. Intriguingly, acti- vation of JAK1 by interleukin-3 also led to phosphorylation of JAK2, suggesting that both JAK1 and JAK2 may be involved in withdrawal signaling. ${ }^{51}$ These findings provide a biological rationale for why inflammation and discontinuation of ruxolitinib could interact to cause more severe withdrawal phenomena.

\section{Ruxolitinib wash-out and rechallenge}

A number of cases have been reported in which a 'drug holiday' led to restoration of ruxolitinib response upon rechallenge, ${ }^{70,71}$ as predicted by the experimental models of ruxolitinib persistence and wash-out. When ruxolitinib is withdrawn the accumulated p-JAK2 becomes susceptible again to ubiquitination and degradation, so that after a wash-out period the untreated cell biology is restored for a variable period of time. Understanding this phenomenon has important implications for the design of clinical trials of second-line agents after ruxolitinib. It is often in the patient's interest to minimize the interval between stopping ruxolitinib and starting a second-line agent, because of the increase in symptom burden that commonly occurs. However, if baseline response assessments for the second-line agent are undertaken too early there may be further deterioration after the 'baseline' assessment. In the randomized FREEDOM-2 study, wash-out and resumption of ruxolitinib will be compared to wash-out of ruxolitinib followed by institution of fedratinib (NCT03952039). This study will provide important new data on the effects of ruxolitinib rechallenge.

\section{Inhibition of JAK1 versus JAK2 versus both}

Many of the symptoms of myelofibrosis are related to increased levels of pro-inflammatory cytokines, including interleukin- 6 and tumor necrosis factor- $\alpha$. Symptomatic improvement in response to ruxolitinib is correlated with a reduction in cytokine levels. ${ }^{72}$ Importantly, many cytokines signal through JAK1 and suppression of JAK1 signaling by dual inhibitors, such as ruxolitinib and momelotinib, may contribute to the symptomatic benefit observed with these treatments. A selective JAK1 inhibitor, itacitinib (INCB039110), has been tested in a phase II clinical trial involving 87 patients with myelofibrosis. $^{73}$ Symptomatic improvement was comparable to that seen with ruxolitinib (total symptom score reduced by $50 \%$ at 24 weeks in $49 \%$ of patients versus $46 \%$ in the COMFORT-I study) whereas the splenic responses were less $(\geq 35 \%$ reduction in spleen volume in $17 \%$ of patients at 24 weeks versus $32-42 \%$ in the COMFORT studies). Conversely, a $50 \%$ reduction in total symptom score was seen in $36 \%$ of patients treated with the JAK2-selective inhibitor, fedratinib. ${ }^{74}$

It may be an oversimplification that JAK1 inhibition mediates improvement in inflammatory symptoms and JAK2 inhibition mediates cytoreductive effects. There may be some crosstalk between JAK1 and JAK2, or splenic responses could perhaps be due to inhibition of cytokinemediated recruitment of cells to sites of extramedullary hematopoiesis.

\section{Secondary resistance to ruxolitinib: comparison with chronic myeloid leukemia}

Secondary resistance to TKI therapy is perhaps best understood in chronic myeloid leukemia. Resistance often 
occurs relatively abruptly and leads to near-complete loss of therapeutic benefit, typically over a number of months. In contrast, loss of response to ruxolitinib in myelofibrosis is characterized by a gradual waning of the initial therapeutic benefit, sometimes associated with late-emerging cytopenia that is likely to represent progression of the underlying disease rather than direct toxicity of the TKI.

In chronic myeloid leukemia, around half of cases of secondary resistance are associated with the emergence of single amino acid changes in the BCR-ABL1 kinase domain which impair binding of the TKI or stabilize the active conformation of the protein. ${ }^{75}$ Ruxolitinib-resistant kinase domain mutations have been isolated using in vitro mutagenesis screening, and such mutations have also been observed clinically in patients with acute lymphoblastic leukemia treated with ruxolitinib. ${ }^{76}$ However, fewer than $50 \%$ of resistant clones after saturation mutagenesis in the presence of ruxolitinib harbor additional mutations in JAK2. ${ }^{49}$ Fedratinib, another ATP-competitive JAK2 inhibitor in clinical use, appears to be less susceptible to this pattern of resistance because it inhibits not only ATP binding, but also the binding of peptide substrates to JAK2." However, JAK2 kinase domain mutations have not yet been observed in clinical samples from myelofibrosis patients treated with JAK inhibitors, which could indicate either incomplete inhibition of JAK2 or that myelofibrosis is not critically dependent on the kinase activity of JAK2 in the way that chronic myeloid leukemia is addicted to a fusion oncogene.

\section{Strategies to improve inhibition of JAK-STAT signaling}

Here we discuss various approaches that might be employed to achieve more complete inhibition of JAKSTAT signaling (summarized in Table 1) with the aim of improving treatment response, and ultimately finding treatments with greater disease-modifying potential. Type 2 kinase inhibitors, such as the BCR-ABL1 inhibitor, imatinib, are ATP-competitive inhibitors that bind the inactive conformation of the kinase. All of the JAK2 inhibitors that have been tested clinically to date for myelofibrosis are type 1 inhibitors. Since JAK2 V617F is a weak activating mutation (relative to constitutive activation of fusion kinases, such as BCR-ABL1 and PCM1-JAK2) it does not strongly favor the active conformation. In contrast, the KIT D816V mutation that is commonly found in systemic mastocytosis leads to a bias in favor of the active conformation of the stem cell factor receptor (which KIT encodes). Midostaurin and avapritinib (both type 1 inhibitors) have significant clinical activity against KIT D816: they show greater potency against KIT D816V than against wild-type KIT, whereas the converse is true for type 2 inhibitors, such as imatinib and sunitinib. ${ }^{78}$ An experimental type 2 JAK inhibitor, CHZ868, did not result in accumulation of p-JAK2 and led to more potent suppression of myelofibrosis cells. 49,51 This compound is not being developed for clinical use, but these pre-clinical data suggest that the development of type 2 JAK inhibitors for clinical use might offer advantages over the available TKI for myelofibrosis. A potential risk of this approach is greater suppression of normal hematopoiesis.

The JH2 pseudokinase domain of JAK2 binds ATP, and mutation of certain residues abrogates ATP binding, as well as leading to loss of the activated JH1 tyrosine kinase activity in V617F.79 In a mouse model of JAK2 V617F MPN, co-mutation with K581A prevented the development of polycythemia. Notably, mutation of the same residue had little or no effect on cytokine signaling through wild-type JAK2, potentially opening the opportunity for the development of mutation-specific allosteric inhibitors that target this site.

JAK2 is a client protein of Hsp90, and inhibition of Hsp90 leads to degradation of JAK2 (including p-JAK2). Combining ruxolitinib with an Hsp90 inhibitor was more

Table 1. Possible strategies to inhibit JAK2 and downstream signaling in myeloproliferative neoplasms.

\begin{tabular}{|c|c|c|c|}
\hline & Mechanism of action & Examples & Notes \\
\hline \multicolumn{4}{|c|}{ Current standard of care } \\
\hline Type 1 inhibitors & $\begin{array}{l}\text { ATP-competitive inhibitors that bind the } \\
\text { active conformation of JAK2 }\end{array}$ & $\begin{array}{l}\text { Ruxolitinib } \\
\text { Fedratinib } \\
\text { Momelotinib } \\
\text { Pacritinib }\end{array}$ & $\begin{array}{l}\text { Lead to accumulation of p-JAK2 } \\
\text { Limited disease-modifying potential }\end{array}$ \\
\hline
\end{tabular}

\begin{tabular}{llll}
$\begin{array}{l}\text { Alternative JAK inhibitors } \\
\text { Type } 2 \text { inhibitors }\end{array}$ & $\begin{array}{l}\text { ATP-competitive inhibitors that bind } \\
\text { the active conformation of JAK2 } \\
\text { Non-ATP-competitive inhibitors of } \\
\text { the pseudokinase domain or substrate binding }\end{array}$ & $\begin{array}{l}\text { CHZ868 } \\
\text { BBT594 } \\
\text { LS104 }\end{array}$ & $\begin{array}{l}\text { Do not lead to accumulation of p-JAK2 } \\
\text { Could confer greater specificity for JAK2 } \\
\text { V617F over wild-type JAK2 }\end{array}$ \\
\hline $\begin{array}{l}\text { Drugs to degrade JAK2 } \\
\text { Hsp90 inhibitors }\end{array}$ & $\begin{array}{l}\text { Inhibit chaperone function to expose } \\
\text { JAK2 to degradation }\end{array}$ & AUY922 & $\begin{array}{l}\text { Clinical development discontinued } \\
\text { because of ocular toxicity } \\
\text { Could reduce accumulation of p-JAK2 } \\
\text { De-ubiquitinase inhibitors }\end{array}$ \\
$\begin{array}{l}\text { Small molecules that promote } \\
\text { ubiquitination without specificity for JAK2 }\end{array}$ & WP1130 & $\begin{array}{l}\text { Could reduce accumulation of p-JAK2 } \\
\text { in combination with a type 1 JAK2 inhibitor }\end{array}$ \\
$\begin{array}{l}\text { Proteolysis-activating chimed to target specific proteins } \\
\text { (PROTAC) } \\
\text { Inhibitors of downstream signaling } \\
\text { MEK/ERK inhibitors }\end{array}$ & $\begin{array}{l}\text { Inhibitors of bypass signaling in } \\
\text { the presence of JAK inhibitor }\end{array}$ & $\begin{array}{l}\text { Synergistic with type 1 JAK2 inhibitor } \\
\text { in experimental models }\end{array}$ \\
\hline
\end{tabular}

Approaches that have been used clinically (in any disease) are shown in bold. 
effective than ruxolitinib monotherapy in a mouse model of myelofibrosis. ${ }^{80}$ A clinical trial using the Hsp90 inhibitor AUY922 was terminated prematurely because of safety concerns, despite evidence of splenic responses. ${ }^{81}$ Other studies using the same drug documented a high frequency of visual alterations, including night blindness. ${ }^{82}$ An alternative approach to target the accumulation of p-JAK2 is to use a proteolysis-targeting chimera (PROTAC). These smallmolecule drugs contain two functional domains: one binds the target protein and the second engages an E3 ubiquitin ligase, thereby triggering proteasomal degradation of the target protein. JAK family PROTAC have been developed that recruit inhibitor of apoptosis protein leading to proteasomal degradation. ${ }^{83}$ An inhibitor of the de-ubiquitinase, USP9X, has been shown to accelerate the degradation of JAK2 in vitro. Whether any of these approaches could selectively target pathological accumulation of p-JAK2 in patients with myelofibrosis remains to be tested.

One of the pathways activated downstream of JAK2 in MPN is RAS/RAF/MEK/ERK. Ruxolitinib treatment of a cell line expressing JAK2 V617F inhibited downstream phosphorylated ERK1 and ERK2 (p-ERK1/2), but ruxolitinib treatment of a V617F mouse MPN model did not, despite effective suppression of other proteins downstream of JAK2 ${ }^{46}$ Persistence of MEK/ERK signaling in vivo was found to be due to activation of PDGFRA by PDGF$\mathrm{BB}$ signaling as a bypass pathway that enables persistence of MPN cells despite effective inhibition of JAK2 kinase activity. Combined inhibition of JAK2 and MEK with ruxolitinib and binimetinib (an approved treatment for RAFmutant melanoma) was more efficient than either TKI alone in achieving regression of splenomegaly, fibrosis and JAK2 V617F allele burden. ${ }^{46}$

\section{Conclusion}

Despite the symptomatic benefits of currently available JAK2 inhibitors, there is a need for agents that have a greater effect on the disease clone and its natural history. JAK2 remains a crucial target in myelofibrosis, and deletion of JAK2 in a mouse model of myelofibrosis substan- tially abrogates disease manifestations. Data from several groups have shown that a conformational change in JAK2 induced by the binding of ruxolitinib and other type 1 inhibitors may contribute to disease persistence by preventing JAK2 de-phosphorylation and proteasomal degradation, allowing heterodimerization of JAK2 with JAK1 or TYK2. Type 2 inhibitors of JAK2 do not cause this phenomenon and have greater impact on disease biology in a mouse model. The V617F mutation leads to a highly inflexible substitution, which may be indicative of a neomorphic change in function, rather than non-specific overactivation. Several lines of evidence suggest that it may be possible to exploit the unique properties of the mutant pseudokinase to develop mutation-specific JAK2 inhibitors that spare normal hematopoiesis. Whereas current clinical trials are mostly targeting cooperating pathways or pathways downstream of JAK2, these observations suggest that novel approaches to the targeting of JAK2 could lead to substantial benefits for patients with myelofibrosis.

\section{Disclosures}

$D M R$ has received research funding and honoraria from Novartis and BMS/Celgene unrelated to the current work. JJB, $D T v$ and DTh declare that they have no conflicts of interest.

\section{Contributions}

$D M R$ and DTh reviewed the literature and wrote the paper. $J J B$ and DTV critically reviewed the content and wrote the paper.

\section{Acknowledgments}

We thank Suraiya Onnesha for assistance with the figures.

\section{Funding}

Research support for DMR was provided through the Medical Research Future Fund and The Hospital Research Foundation. Research support for JJB was provided by the National Health and Medical Research Council (APP1113577; APP1121755; APP1122999). Research support for DTh was provided through the National Health and Medical Research Council (APP1182564), Medical Research Future Fund, The Hospital Research Foundation and a CSL Centenary Fellowship.

\section{References}

1. Wernig G, Mercher T, Okabe R, Levine RL, Lee BH, Gilliland DG. Expression of Jak2V617F causes a polycythemia vera-like disease with associated myelofibrosis in a murine bone marrow transplant model. Blood. 2006;107(11):4274-4281.

2. Nangalia J, Massie CE, Baxter EJ, et al. Somatic CALR mutations in myeloproliferative neoplasms with nonmutated JAK2. N Engl J Med. 2013;369(25):2391-2405

3. Reinisch A, Thomas D, Corces MR, et al. A humanized bone marrow ossicle xenotransplantation model enables improved engraftment of healthy and leukemic human hematopoietic cells. Nat Med. 2016;22(7): 812-821.

4. Baxter EJ, Scott LM, Campbell PJ, et al. Acquired mutation of the tyrosine kinase JAK2 in human myeloproliferative disorders. Lancet. 2005;365(9464):1054-1061.

5. Kralovics R, Passamonti F, Buser AS, et al. A gain-of-function mutation of JAK2 in myeloproliferative disorders. $N$ Engl J Med.
2005:352(17):1779-1790

6. Hughes TP, Kaeda J, Branford S, et al. Frequency of major molecular responses to imatinib or interferon alfa plus cytarabine in newly diagnosed chronic myeloid leukemia. N Engl J Med. 2003;349(15):1423-1432.

7. Kantarjian HM, Hochhaus A, Saglio G, et al. Nilotinib versus imatinib for the treatment of patients with newly diagnosed chronic phase, Philadelphia chromosome-positive, chronic myeloid leukaemia: 24-month minimum follow-up of the phase 3 randomised ENESTnd trial. Lancet Oncol. 2011;12(9): 841-851.

8. Cortes JE, Saglio G, Kantariian HM, et al. Final 5-year study results of DASISION: the Dasatinib Versus Imatinib Study in Treatment-Naive Chronic Myeloid Leukemia Patients trial. J Clin Oncol. 2016;34(20):2333-2340.

9. Stark GR, Darnell JE Jr. The JAK-STAT pathway at twenty. Immunity. 2012;36(4):503514.

10. Liau NPD, Laktyushin A, Morris R, et al. Enzymatic characterization of wild-type and mutant Janus kinase 1. Cancers (Basel).
2019;11(11):1701

11. Kershaw NJ, Murphy JM, Liau NP, et al. SOCS3 binds specific receptor-JAK complexes to control cytokine signaling by direct kinase inhibition. Nat Struct Mol Biol. 2013;20(4):469-476.

12. Kershaw NJ, Murphy JM, Lucet IS, Nicola NA, Babon JJ. Regulation of Janus kinases by SOCS proteins. Biochem Soc Trans. 2013;41(4):1042-1047.

13. Liau NPD, Laktyushin A, Lucet IS, et al. The molecular basis of JAK/STAT inhibition by SOCS1. Nat Commun. 2018;9(1):1558.

14. Lv K, Jiang J, Donaghy R, et al. CBL family E3 ubiquitin ligases control JAK2 ubiquitination and stability in hematopoietic stem cells and myeloid malignancies. Genes Dev. 2017;31(10):1007-1023.

15. Chou DH, Vetere A, Choudhary A, et al. Kinase-independent small-molecule inhibition of JAK-STAT signaling. J Am Chem Soc. 2015;137(24):7929-7934.

16. Grinfeld J, Nangalia J, Baxter EJ, et al. Classification and personalized prognosis in myeloproliferative neoplasms. N Engl J Med. 2018;379(15):1416-1430. 
17. Lu X, Huang LJ, Lodish HF. Dimerization by a cytokine receptor is necessary for constitutive activation of JAK2V617F. J Biol Chem. 2008;283(9):5258-5266

18. Lu X, Levine R, Tong W, et al. Expression of a homodimeric type I cytokine receptor is required for JAK2V617F-mediated transformation. Proc Natl Acad Sci U S A. 2005;102 (52):18962-18967.

19. Ungureanu $\mathrm{D}, \mathrm{Wu}$ J, Pekkala $\mathrm{T}$, et al. The pseudokinase domain of JAK2 is a dualspecificity protein kinase that negatively regulates cytokine signaling. Nat Struct Mol Biol. 2011;18(9):971-976.

20. Jeong EG, Kim MS, Nam HK, et al. Somatic mutations of JAK1 and JAK3 in acute leukemias and solid cancers. Clin Cancer Res. 2008;14(12):3716-3721.

21. Staerk J, Kallin A, Demoulin JB, Vainchenker W, Constantinescu SN. JAK1 and Tyk2 activation by the homologous polycythemia vera JAK2 V617F mutation: cross-talk with IGF1 receptor. J Biol Chem. 2005;280(51): 41893-41899.

22. Wilmes S, Hafer M, Vuorio J, et al. Mechanism of homodimeric cytokine receptor activation and dysregulation by oncogenic mutations. Science. 2020;367(6478): 643-652.

23. Moliterno AR, Williams DM, Rogers O, Isaacs MA, Spivak JL. Phenotypic variability within the JAK2 V617F-positive MPD: roles of progenitor cell and neutrophil allele burdens. Exp Hematol. 2008;36(11):1480-1486.

24. Stein BL, Saraf S, Sobol U, et al. Age-related differences in disease characteristics and clinical outcomes in polycythemia vera. Leuk Lymphoma. 2013;54(9):1989-1995.

25. Gangat N, Tefferi A. Myelofibrosis biology and contemporary management. Br J Haematol. 2020:191(2):152-170.

26. Ortmann CA, Kent DG, Nangalia J, et al. Effect of mutation order on myeloproliferative neoplasms. N Engl J Med. 2015;372(7): 601-612.

27. Klampfl T, Gisslinger H, Harutyunyan AS, et al. Somatic mutations of calreticulin in myeloproliferative neoplasms. N Engl J Med. 2013;369(25):2379-2390.

28. Eder-Azanza L, Navarro D, Aranaz P, Novo FJ, Cross NC, Vizmanos JL. Bioinformatic analyses of CALR mutations in myeloproliferative neoplasms support a role in signaling. Leukemia. 2014;28(10):2106-2109.

29. Tefferi A, Lasho TL, Finke C, et al. Type 1 vs type 2 calreticulin mutations in primary myelofibrosis: differences in phenotype and prognostic impact. Leukemia. 2014;28(7): 1568-1570

30. Guglielmelli P, Lasho TL, Rotunno G, et al. MIPSS70: Mutation-enhanced International Prognostic Score System for transplantationage patients with primary myelofibrosis. J Clin Oncol. 2018;36(4):310-318.

31. Clinton A, McMullin MF. The calreticulin gene and myeloproliferative neoplasms. J Clin Pathol. 2016;69(10):841-845.

32. Elf S, Abdelfattah NS, Baral AJ, et al. Defining the requirements for the pathogenic interaction between mutant calreticulin and MPL in MPN. Blood. 2018;131(7):782-786.

33. Elf S, Abdelfattah NS, Chen E, et al. Mutant calreticulin requires both its mutant $\mathrm{C}$-terminus and the thrombopoietin receptor for oncogenic transformation. Cancer Discov. 2016;6(4):368-381

34. Pecquet C, Chachoua I, Roy A, et al. Calreticulin mutants as oncogenic rogue chaperones for TpoR and traffic-defective pathogenic TpoR mutants. Blood. 2019;133 (25):2669-2681

35. Sollazzo D, Forte D, Polverelli N, et al.
Circulating calreticulin is increased in myelofibrosis: correlation with interleukin-6 plasma levels, bone marrow fibrosis, and splenomegaly. Mediators Inflamm. 2016; 2016:5860657.

36. Pecquet C, Balligand T, Chachoua I, et al. Secreted mutant calreticulins as rogue cytokines trigger thrombopoietin receptor activation specifically in CALR mutated cells: perspectives for MPN therapy. Blood. 2018;132(Suppl 1):4.

37. Guglielmelli P, Rotunno G, Bogani C, et al Ruxolitinib is an effective treatment for CALR-positive patients with myelofibrosis. Br J Haematol. 2016;173(6):938-940.

38. Verstovsek S, Mesa RA, Gotlib J, et al. A double-blind, placebo-controlled trial of ruxolitinib for myelofibrosis. N Engl J Med. 2012;366(9):799-807.

39. Harrison C, Kiladjian JJ, Al-Ali HK, et al. JAK inhibition with ruxolitinib versus best available therapy for myelofibrosis. $\mathrm{N}$ Engl J Med. 2012;366(9):787-798.

40. Verstovsek S, Mesa RA, Gotlib J, et al. The clinical benefit of ruxolitinib across patient subgroups: analysis of a placebo-controlled, phase III study in patients with myelofibrosis. Br J Haematol. 2013;161(4):508-516.

41. Godfrey AL, Chen E, Pagano F, et al. JAK2V617F homozygosity arises commonly and recurrently in PV and ET, but PV is characterized by expansion of a dominant homozygous subclone. Blood. 2012;120(13): 2704-2707.

42. Cervantes F, Vannucchi AM, Kiladjian JJ, et al. Three-year efficacy, safety, and survival findings from COMFORT-II, a phase 3 study comparing ruxolitinib with best available therapy for myelofibrosis. Blood. 2013;122(25):4047-4053.

43. Harrison CN, Vannucchi AM, Kiladjian JJ, et al. Long-term findings from COMFORT-II, a phase 3 study of ruxolitinib vs best available therapy for myelofibrosis. Leukemia. 2016;30(8):1701-1707.

44. Masarova L, Wang W, Newberry KJ Kantarjian H, Verstovsek S. Complete remission in a patient with JAK2- and IDH2-positive myelofibrosis. Blood. 2016;128(6):877880.

45. Verstovsek S, Gotlib J, Mesa RA, et al. Longterm survival in patients treated with ruxolitinib for myelofibrosis: COMFORT-I and -II pooled analyses. J Hematol Oncol. 2017;10(1):156.

46. Stivala S, Codilupi T, Brkic S, et al. Targeting compensatory MEK/ERK activation increases JAK inhibitor efficacy in myeloproliferative neoplasms. J Clin Invest. 2019;129(4): 1596-1611.

47. Nangalia J, Nice FL, Wedge DC, et al. DNMT3A mutations occur early or late in patients with myeloproliferative neoplasms and mutation order influences phenotype. Haematologica. 2015;100(11):e438-442.

48. Hatzivassiliou G, Song K, Yen I, et al. RAF inhibitors prime wild-type RAF to activate the MAPK pathway and enhance growth Nature. 2010;464(7287):431-435.

49. Koppikar P, Bhagwat N, Kilpivaara O, et al. Heterodimeric JAK-STAT activation as mechanism of persistence to JAK2 inhibitor therapy. Nature. 2012:489(7414):155-159.

50. Andraos R, Qian Z, Bonenfant D, et al. Modulation of activation-loop phosphorylation by JAK inhibitors is binding mode dependent. Cancer Discov. 2012;2(6):512 523 .

51. Tvorogov D, Thomas D, Liau NPD, et al Accumulation of JAK activation loop phosphorylation is linked to type I JAK inhibito withdrawal syndrome in myelofibrosis. Sci Adv. 2018;4(11):eaat3834.

52. Bankar A, Gupta V. Investigational non-JAK inhibitors for chronic phase myelofibrosis. Expert Opin Investig Drugs. 2020;29(5):461 474.

53. Haan S, Wuller S, Kaczor J, et al. SOCS mediated downregulation of mutant Jak2 (V617F, T875N and K539L) counteracts cytokine-independent signaling. Oncogene. 2009;28(34):3069-3080

54. Grandage VL, Everington T, Linch DC, Khwaja A. Go6976 is a potent inhibitor of the JAK 2 and FLT3 tyrosine kinases with significant activity in primary acute myeloid leukaemia cells. Br J Haematol. 2006;135 (3):303-316

55. Marty C, Pecquet C, Nivarthi $\mathrm{H}$, et al Calreticulin mutants in mice induce an MPLdependent thrombocytosis with frequent progression to myelofibrosis. Blood 2016:127(10):1317-1324.

56. Balligand T, Achouri Y, Pecquet C, et al. Pathologic activation of thrombopoietin receptor and JAK2-STAT5 pathway by frameshift mutants of mouse calreticulin Leukemia. 2016;30(8):1775-1778.

57. Nivarthi $\mathrm{H}$, Chen D, Cleary C, et al Thrombopoietin receptor is required for the oncogenic function of CALR mutants. Leukemia. 2016;30(8):1759-1763.

58. Shi JG, Chen X, McGee RF, et al. The pharmacokinetics, pharmacodynamics, and safety of orally dosed INCB018424 phosphate in healthy volunteers. J Clin Pharmacol. 2011;51(12):1644-1654

59. Zhang M, Xu C, Ma L, et al. Effect of food on the bioavailability and tolerability of the JAK2-selective inhibitor fedratinib (SAR302503): results from two phase I studies in healthy volunteers. Clin Pharmacol Drug Dev. 2015;4(4):315-321

60. Younes A, Romaguera J, Fanale $M$ et al. Phase I study of a novel oral Janus kinase 2 inhibitor, SB1518, in patients with relapsed lymphoma: evidence of clinical and biologic activity in multiple lymphoma subtypes. J Clin Oncol. 2012;30(33):4161-4167.

61. Pardanani A, Laborde RR, Lasho TL, et al Safety and efficacy of CYT387, a JAK1 and JAK2 inhibitor, in myelofibrosis. Leukemia. 2013;27(6):1322-1327.

62. Tefferi A, Pardanani A. Serious adverse events during ruxolitinib treatment discontinuation in patients with myelofibrosis. Mayo Clin Proc. 2011;86(12):1188-1191

63. Coltro G, Mannelli F, Guglielmelli P, Pacilli A, Bosi A, Vannucchi AM. A life-threatening ruxolitinib discontinuation syndrome. Am J Hematol. 2017;92(8):833-838.

64. Dai T, Friedman EW, Barta SK. Ruxolitinib withdrawal syndrome leading to tumor lysis. J Clin Oncol. 2013;31(29):e430-432.

65. Beauverd Y, Samii K. Acute respiratory distress syndrome in a patient with primary myelofibrosis after ruxolitinib treatment discontinuation. Int J Hematol. 2014;100(5): 498-501.

66. Herman DD, Kempe CB, Thomson CC McCallister JW. Recurrent hypoxemic respiratory failure. Beyond the usual suspects. Ann Am Thorac Soc. 2014;11(7):1145-1148.

67. Harrison C, Mesa R, Ross D, et al. Practical management of patients with myelofibrosis receiving ruxolitinib. Expert Rev Hematol. 2013;6(5):511-523.

68. National Comprehensive Cancer Network NCCN Clinical Practice Guidelines in Oncology: Myeloproliferative Neoplasms. 2020 [accessed August 5, 2020]

69. Palandri F, Breccia M, Bonifacio M, et al. Life 
Persistence of myelofibrosis treated with ruxolitinib

after ruxolitinib: reasons for discontinuation, impact of disease phase, and outcomes in 218 patients with myelofibrosis. Cancer. 2020;126(6):1243-1252.

70. Gisslinger $\mathrm{H}$, Schelling $\mathrm{M}$, Gisslinger $\mathrm{B}$, Skrabs C, Mullauer L, Kralovics R. Restoration of response to ruxolitinib upon brief withdrawal in two patients with myelofibrosis. Am J Hematol. 2014;89(3): 344-346.

71. Girds A, Bu D, Martynova A, et al. Ruxolitinib rechallenge can improve constrtuitional symptoms and splenomegaly in patients with myelofibrosis: a case series. Chin Lymphoma Myeloma Leek. 2018;18 (11):e463-e468.

72. Verstovsek S, Kantarjian H, Mesa RA, et al. Safety and efficacy of INCB018424, a JAK1 and JAK2 inhibitor, in myelofibrosis. N Angl J Med. 2010;363(12):1117-1127.

73. Mascarenhas JO, Talpaz M, Gupta V, et al. Primary analysis of a phase II open-label trial of INCB039110, a selective JAK1 inhibitor, in patients with myelofibrosis. Haematological. 2017;102(2):327-335.

74. Pardanani A, Harrison C, Cortes JE, et al. Safety and efficacy of fedratinib in patients with primary or secondary myelofibrosis: a randomized clinical trial. JAMA Oncol. 2015;1(5):643-651.

75. Soverini S, Colarossi S, Gnani A, et al. Contribution of ABL kinase domain mutatons to imatinib resistance in different subsets of Philadelphia-positive patients: by the GIMEMA Working Party on Chronic Myeloid Leukemia. Chin Cancer Res. 2006;12(24):7374-7379.

76. Sudras T, Heatley SL, Rok CH, et al. A novel somatic JAK2 kinase-domain mutation in pediatric acute lymphoblastic leukemia with rapid on-treatment development of $\mathrm{LOH}$. Cancer Genet. 2017;216-217:86-90.

77. Kesarwani M, Huber E, Kincaid Z, et al. Targeting substrate-site in Jake kinase provents emergence of genetic resistance. Sci Rep. 2015;5:14538.

78. Evans EK, Gardino AK, Kim JL, et al. A procision therapy against cancers driven by KIT/PDGFRA mutations. Sci Transl Med. 2017;9(414):eaao1690.

79. Hammaren HM, Ungureanu D, Grisouard J, Skoda RC, Hubbard SR, Silvennoinen O. ATP binding to the pseudokinase domain of JAK2 is critical for pathogenic activation. Proc Natl Accad Sci U S A. 2015;112(15): 4642-4647.

80. Bhagwat N, Koppikar P, Keller M, et al.
Improved targeting of JAK2 leads to increased therapeutic efficacy in myeloproliferative neoplasms. Blood. 2014;123(13): 2075-2083.

81. Hobbs GS, Hanasoge Somasundara AV, Kleppe M, et al. Hsp90 inhibition disrupts JAK-STAT signaling and leads to reductions in splenomegaly in patients with myeloproliferative neoplasms. Haematological. 2018;103(1):e5-e9.

82. Johnson ML, Yo HA, Hart EM, et al. Phase I/II study of HSP90 inhibitor AUY922 and erlotinib for EGFR-mutant lung cancer with acquired resistance to epidermal growth factor receptor tyrosine kinase inhibitors. J Chin Oncol. 2015;33(15):1666-1673.

83. Shah RR, Redmond JM, Mihut A, et al. HiJAK-ing the ubiquitin system: the design and physicochemical optimisation of JAK PROTACs. Bioorg Med Chem. 2020;28(5): 115326.

84. Akiyama H, Umezawa Y, Watanabe D, et al. Inhibition of USP9X downregulates JAK2V617F and induces apoptosis synergistically with $\mathrm{BH} 3$ mimetic preferentially in ruxolitinib-persistent JAK2-V617F-positive leukemic cells. Cancers (Basel). 2020;12(2): 406.

haematological | 2021; 106(5)

1253 